ИЗВЕСТИЯ АКАДЕМИИ НАУК ЭСТОНСКОП ССР. ТОМ 26 ФИЗИКА * МАТЕМАТИКА. 1977, № 4

\title{
АНАЛИЗ ТИРИСТОРНОГО РЕГУЛЯТОРА ПЕРЕМЕННОГО НАПРЯЖЕНИЯ С ПОДАВЛЕННЫМИ ЧЕТНЫМИ ГАРМОНИКАМИ ПРИ АКТИВНО-ИНДУКТИВНОЙ НАГРУЗКЕ
}

Одним из недостатков широко распространенных тиристорных регуляторов переменного напряжения является генерирование нежелательных новых частот, искажающих выходное напряжение и ток питания. Перспективным способом улучшения спектрального состава тока и напряжения регулятора является подавление четных гармоник в токе тиристора $\left[{ }^{1-4}\right]$. В случае схемы регулятора переменного напряжения со встречно-параллельно включенными тиристорами этот способ реализуется включением в контур тиристорной пары линейного дросселя Д со средней точкой. Работа такой схемы анализировалась только при активной нагрузке $\left[{ }^{3,4}\right]$. Цель настоящей статьи состоит в изучении работы схемы в более общем случае - при активно-индуктивной нагрузке.

На рис. 1 изображена принципиальная схема анализируемого регулятора, нагрузкой которого служат последовательно включенные активное сопротивление $R$ и индуктивность $L$.

Индуктивность одной полуобмотки дросселя в контуре тиристорной

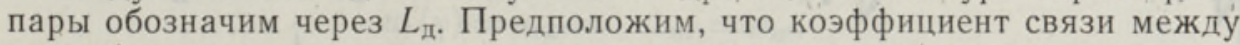
полуобмотками дросселя равен единице и полуобмотки идентичны. Тогда индуктивность обмотки дросселя в целом будет равна $4 L_{\text {д. }}$

Для упрощения анализа работы схемы допустим, что напряжение питания синусоидально, тиристоры Т1 и Т2 идеальны, активное сопротивление в контуре, образованном отпертыми тиристорами и дросселем, равно нулю и индуктивность $L_{д}$ бесконечно большая.

В работе схемы за полупериод различаются два этапа: коммутационный и внекоммутационный. Длительность коммутационного этапа обозначим через $\gamma$. В течение $\gamma$ открыты оба тиристора и падения напряжения на дросселе нет, так как существует короткозамкнутый контур, для которого справедливо

$$
\begin{gathered}
4 L_{\text {д }} \mathrm{d} i_{0} / \mathrm{d} t=0, \\
i_{0}=\text { const, }
\end{gathered}
$$

где $i_{0}-$ фиктивный ток, циркулирующий в контуре.

Ток $i_{0}$ отражает постоянство намагничивающей силы дросселя в анализируемом идеализированном регуляторе. При сделанных предположениях о бесконечности индуктивности дросселя (и, соответственно, о бесконечности накопленной в дросселе энергии!), а также об отсутствии активного сопротивления в контуре тиристорной пары, намагничивающая сила дросселя запџишетст в виде 


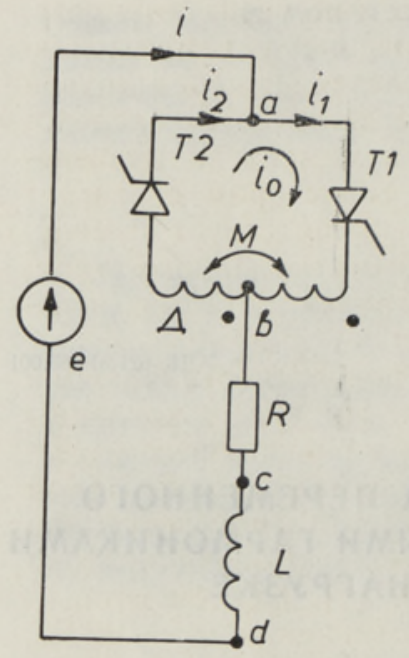

Рис . 1. Принципиальная схема регулятора переменного напряжения.
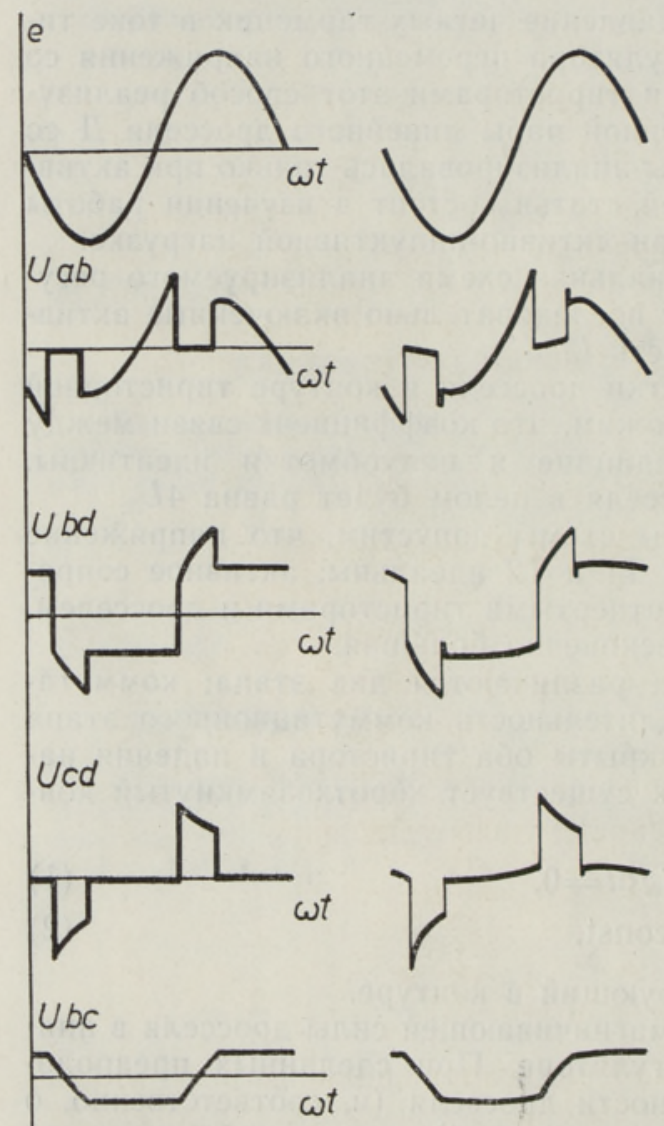

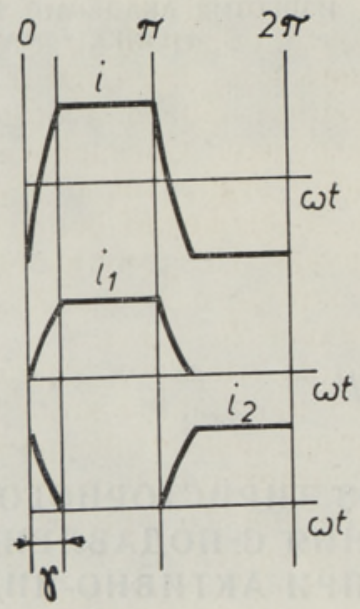

Рис. 2. Временные диаграммы токов $i, i_{1}, i_{2}$.

$$
F=i_{1} w+i_{2} w=2 i_{0} w=\text { const, }
$$

где $w-$ число витков полуобмотки дросселя; $i_{1}$ и $i_{2}-$ токи через тиристоры Т1 и Т2 соответственно.

Таким образом, фиктивный ток циркуляции равен

$$
\begin{aligned}
i_{0}= & \frac{i_{1}+i_{2}}{2}=i_{2}-\frac{i}{2}= \\
& =i_{1}+\frac{i}{2}=\text { const. }
\end{aligned}
$$

Здесь $i=i_{2}-i_{1}-$ ток нагрузки, для которого справедливо дифференциальное уравнение

$L \mathrm{~d} i / \mathrm{d} t+i R=E_{m} \sin (\omega t+\alpha)$,

где $\alpha-$ угол отпирания тиристоров, отсчитываемый от начала синусоиды $E_{m} \sin \omega t$. Согласно формуле (5), время отсчитывается с момента открывания вентиля. Временные диаграммы токов $i, i_{1}$, $i_{2}$ изображены на рис 2.

Рис. 3. Диаграммы мгновенных значений напряжений на различных участках схемы: $a-$ теоретические, $\sigma-$ осциллограммы. 
Коммутационный этап кончается тогда, когда ток через тиристор, который включен обратно по отношению к мгновенному напряжению сети, уменьшается до нуля. Этот тиристор запирается, и через другой открытый тиристор одна полуобмотка дросселя Д включается последовательно с нагрузкой. Согласно выражению (4), в момент начала внекоммутационного этапа ток в ветви с открытым тиристором достигает величины $2 i_{0}$. Бесконечно большое значение $L_{д}$ препятствует изменению тока нагрузки. Поэтому в течение всего внекоммутационного этапа, длительность которого равна $\lambda=\pi-\gamma$, ток нагрузки остается неизменным и равным $2 i_{0}$.

Как только на электрод запертого тиристора подается отпирающий импульс, снова начинается коммутационный этап. На рис. 3 показаны теоретические временные диаграммы напряжения на различных участках схемы и соответствующие этим напряжениям осциллограммы. Из-за конечного значения $L_{\text {д }}$ кривые, снятые экспериментально, несколько отличаются от теоретических. Для расчета мгновенного значения тока нагрузки применим метод относительных величин $\left[{ }^{5}\right]$. Введем относительную величину напряжения на активном сопротивлении $u$ * $=i R / E_{m}$, электромагнитную постоянную времени нагрузки $T_{\text {э }}=L / R$ и соответственно фазовый угол нагрузки $\Theta=\operatorname{arctg}(\omega L / R)$. Теперь уравнение (5) запишется в виде

$$
T_{\text {э }} \mathrm{d} u_{*} / \mathrm{d} t+u_{*}=\sin (\omega t+\alpha) .
$$

Решив соотношение (5а) относительно $u$, получим

$$
\begin{gathered}
u_{*}=\left[u_{\mathrm{H} 1^{*}}-\cos \Theta \sin (\alpha-\Theta)\right] \exp (-\omega t \operatorname{ctg} \Theta)+ \\
+\cos \Theta \sin (\omega t+\alpha-\Theta) .
\end{gathered}
$$

Здесь $u_{\mathrm{H} 1^{*}}=u_{*}(0)$ - начальное условие. Если нам известны значения величин $u_{\text {н1* }}$ и $\gamma$, то, подставив в формулу (6) $u_{\mathrm{H} 1 *}$ и $\omega t=\gamma$, найдем значение $u$ * в конце коммутационного этапа $u_{*}(\gamma)=u_{\text {н2* }}$. Это значение останется неизменным в течение всего внекоммутационного этапа и явится начальным условием для следующего, коммутационного, т. е.

$$
u *(\gamma)=u_{\mathrm{H} 2^{*}}=-u_{\mathrm{H} 1^{*}}=u_{\mathrm{H}^{*}} .
$$

Изменение величины $u$ * за период описывается функцией

$$
u_{*}=\left\{\begin{array}{lr}
{\left[-u_{\mathrm{H}^{*}}-\cos \Theta \sin (\alpha-\Theta)\right] \exp (-\omega t \operatorname{ctg} \Theta)+\cos \Theta \sin (\omega t+\alpha-\Theta)} \\
u_{\mathrm{H}^{*}} \text { при } 0 \leqslant \omega t \leqslant \gamma, \\
{\left[u_{\mathrm{H}^{*}}+\cos \Theta \sin (\alpha-\Theta)\right] \exp [(\pi-\omega t) \operatorname{ctg} \Theta]-\cos \Theta \sin (\omega t+\alpha-\pi-\Theta)} \\
-u_{\mathrm{H}^{*}} \text { при } \pi \leqslant \omega t \leqslant \pi f \gamma,
\end{array}\right.
$$

Для внекоммутационного этапа справедливо уравнение

$$
L \mathrm{~d} i / \mathrm{d} t+i R+u_{\mathrm{p}}=E_{m} \sin (\omega t+\alpha)
$$

или

$$
T_{3} \mathrm{~d} u_{*} / \mathrm{d} t+u_{*}+u_{\mathrm{p}^{*}}=\sin (\omega t+\alpha),
$$

где $u_{\mathrm{p}^{*}}=u_{\mathrm{p}} / E_{m}$ - относительное значение напряжения на регуляторе, равное относительному значению напряжения на полуобмотке дросселя $u_{д^{*}}=u_{\Omega} / E_{m}$. Учитывая, что в течение внекоммутационного этапа $\mathrm{d} u \cdot / \mathrm{d} t=0 \quad$ и $\quad u_{*}=u_{\mathrm{H}} \operatorname{sign} \sin \omega t$, имеем 


$$
u_{\mathrm{I}^{*}}=u_{\mathrm{p}^{*}}=\sin (\omega t+\alpha)-u_{\mathrm{H}^{*}} \operatorname{sign} \sin \omega t .
$$

Так как на дросселе не может быть постоянной составляющей падения напряжения, то из условия

$$
\int_{v}^{\pi} u_{\text {म }^{*}} \mathrm{~d} \omega t=0
$$

следует

$$
\cos \alpha+\cos (\alpha+\gamma)-u_{\mathrm{H}^{*}}(\pi-\gamma)=0 .
$$

Подставив в уравнение (6) $\omega t=\gamma$, получим выражение для начального условия

$$
u_{\mathrm{H}^{*}}=\frac{\cos \Theta[\sin (\alpha+\gamma-\Theta)-\sin (\alpha-\Theta) \exp (-\gamma \operatorname{ctg} \Theta)]}{1+\exp (-\gamma / \operatorname{tg} \Theta)},
$$

подстановка которого в уравнение (11) даст трансцендентное уравнение для определения $\gamma$ при заданных $\alpha$ и $\Theta$ :

$$
\begin{gathered}
\cos \alpha+\cos (\alpha+\gamma)-(\pi-\gamma) \times \\
\times \frac{\cos \Theta[\sin (\alpha+\gamma-\Theta)-\sin (\alpha-\Theta) \exp (-\gamma \operatorname{ctg} \Theta)]}{1+\exp (-\gamma \operatorname{ctg} \Theta)}=0 .
\end{gathered}
$$

Зная $\gamma$ и $u_{\mathrm{H}^{*}}$ при заданных $\alpha$ и. $\Theta$, можно из выражения (7) найти значения $u_{*}$, а действующее значение $u_{*}$ за период определить из формулы

$$
u_{* 0}=\sqrt{\frac{1}{2 \pi} \int_{0}^{2 \pi}[u *(\omega t)]^{2} \mathrm{~d} \omega t=}
$$

$=\sqrt{\frac{1}{2 \pi}\left\{2\left[u_{\mathrm{H}^{*}}+\cos \Theta \sin (\alpha-\Theta)\right][\exp (-\gamma \operatorname{ctg} \Theta) \sin (\alpha-\gamma)-\sin \alpha] \sin 2 \Theta+\right.}$

$$
\begin{aligned}
& \overline{+2 u_{\mathrm{H}^{*}}^{2}(\pi-\gamma)+\left[u_{\mathrm{H}^{*}}+\cos \Theta \sin (\alpha-\Theta)\right]^{2}[1-\exp (-2 \gamma \operatorname{ctg} \Theta)] \operatorname{tg} \Theta+} \\
& \left.+\cos ^{2} \Theta\left[\gamma-1 / 2 \cos 2(\alpha-\Theta) \sin 2 \gamma+\sin 2(\alpha-\Theta) \sin ^{2} \gamma\right]\right\} .
\end{aligned}
$$

Коэффициенты для первой гармоники определяются из выражений $a_{1}=\frac{2}{\pi}\left\{\sin \Theta\left[u_{\mathrm{H}^{*}}+\cos \Theta \sin (\alpha-\Theta)\right][\exp (-\gamma \operatorname{ctg} \Theta) \sin (\alpha+\Theta)-\sin \Theta]+\right.$

$$
\begin{gathered}
+\frac{\cos \Theta}{2}[\cos (\alpha-\Theta)(\gamma-\sin \gamma \cos \gamma)+ \\
\left.\left.+\sin (\alpha-\Theta) \sin ^{2} \gamma\right]+u_{\mathrm{H}^{*}}(\cos \gamma+1)\right\},
\end{gathered}
$$

$b_{1}=\frac{2}{\pi}\left\{\sin \Theta\left[u_{\mathrm{n}^{*}}+\cos \Theta \sin (\alpha-\Theta)\right][\exp (-\gamma \operatorname{ctg} \Theta) \cos (\gamma+\dot{\Theta})-\cos \Theta]+\right.$

$$
\begin{gathered}
+\frac{\cos \Theta}{2}[\sin (\alpha-\Theta)(\gamma+\sin \gamma \cos \gamma)+ \\
\left.\left.+\cos (\gamma-\Theta) \sin ^{2} \gamma\right]-u_{\mathrm{H}^{*}} \sin \gamma\right\} .
\end{gathered}
$$

За счет того, что в контур тиристорной пары включен дроссель Д, 


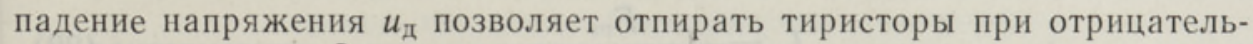
ных значениях $\alpha$. Относительное напряжение на аноде запертого тиристора можно представить в виде

$$
u_{\mathrm{a}}=-2 u_{\mathrm{д}^{*}}=-2\left[\sin (\omega t+\alpha)-u_{\mathrm{H}^{*}} \operatorname{sign} \sin \omega t\right]
$$

При $\omega t=\pi$, т. е. в конце внекоммутационного этапа, в момент посылки импульса на управляющий электрод тиристора получим

$$
u_{\mathrm{a}^{*}}=-2\left(-\sin \alpha-u_{\mathrm{H}^{*}}\right)=2\left(u_{\mathrm{H}^{*}}+\sin \alpha\right) .
$$

Так как тиристор отпирается при $u_{\mathrm{a}^{*}}>0$, то, решив выражение (17a) относительно $\alpha$ при $u_{\mathrm{a}}=0$, найдем такое предельное значение $\alpha=\alpha_{\text {пр }}$, что для всех $\alpha>\alpha_{\text {пр }}$ выполняется условие $u_{\mathrm{a}}$ : $>0$.

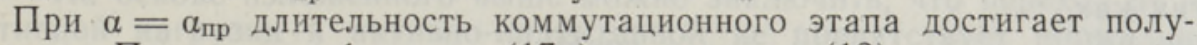
периода. Подставив в формулу (17a) выражение (12) для $u_{\mathrm{H}^{*}}$, приравняв полученное соотношение нулю и приняв $\gamma=\pi$, можем записать

$$
\cos \Theta \sin \left(\alpha_{\mathrm{mp}}-\Theta\right)-\sin \alpha_{\text {пр }}=0 \text {, }
$$

откуда

$$
\alpha_{\mathrm{mp}}=\Theta-\pi / 2 .
$$

Рассмотрим два предельных случая.

1. Нагрузка чисто активная, т. е. $\Theta=0$. Из выражения (7) имеем

$$
u *=\left\{\begin{array}{lll}
\sin (\alpha+\omega t) & \text { при } 0 \leqslant \omega t \leqslant \gamma, \\
u_{\mathrm{H}^{*}} & \text { при } \quad \gamma \leqslant \omega t \leqslant \pi .
\end{array}\right.
$$

Поскольку

$$
u_{\mathrm{H}^{*}}=\sin (\alpha+\gamma) \text {, }
$$

то из (11), выделив $u_{\text {н* }}$ и подставив полученное в выражение (19), найдем

$$
\sin (\alpha+\gamma)=\frac{\cos \alpha+\cos (\alpha+\gamma)}{\pi-\gamma} .
$$

Подставив в равенство (20) значение $\gamma=0$, получим

$$
\alpha=\operatorname{arctg} \frac{2}{\pi},
$$

а из выражения (18) -

$$
\alpha_{\text {пр }}=-\pi / 2 \text {. }
$$

Следовательно, для того чтобы при чисто активной нагрузке получить изменение тока от максимума до нуля, нужно изменять $\alpha$ в пределах от $-\pi / 2$ до $\pi / 2$ эл. град., причем коммутационный этап соответствует только промежутку

$$
-\frac{\pi}{2}<\alpha<\operatorname{arctg} \frac{2}{\pi},
$$

при значениях $\alpha>\operatorname{arctg} 2 / \pi$ длительность коммутационного этапа равна нулю.

2. Нагрузка чисто индуктивная. Величина $u$. обращается в нуль, так как $u$ : $=i R / E_{m}$, а $R=0$. Введем относительную величину тока нагрузки

$$
i *=i / I_{m},
$$

где $I_{m}-$ базовая величина, равная амплитуде тока при $\alpha=\alpha_{\text {пр }}$ 


$$
I_{m}=\frac{E_{m}}{\sqrt{R^{2}+(\omega L)^{2}}} .
$$

После подстановки формулы (22) в уравнение (21) и преобразования получим

$$
i_{*}=u_{*} / \cos \Theta \text {. }
$$

Подстановка $\Theta=\pi / 2$ в условие (12) дает

$$
i_{\mathrm{H}^{*}}=\frac{u_{\mathrm{H}^{*}}}{\cos \Theta}=\frac{\cos \Theta}{\cos \Theta}\left[\frac{\cos \alpha-\cos (\alpha+\gamma)}{2}\right] \text {. }
$$

Так как

$$
\lim _{\Theta \rightarrow \pi / 2} \frac{\cos \Theta}{\cos \Theta}=1
$$

то

$$
i_{\mathrm{H}^{*}}=(\cos \alpha-\cos (\alpha+\gamma)) / 2 .
$$

Из выражений (6) и (21) определим мгновенные значения для $i$ в течение периода:

$i_{*}=\left\{\begin{array}{lll}-i_{\mathrm{H}^{*}}+\cos \alpha-\cos (\alpha+\omega t) & \text { при, } & 0 \leqslant \omega t \leqslant \gamma, \\ i_{\mathrm{H}^{*}} & \text { при } & \gamma \leqslant \omega t \leqslant \pi, \\ i_{\mathrm{H}^{*}}-\cos \alpha+\cos (\alpha+\omega t-\pi) & \text { при } & \pi \leqslant \omega t \leqslant \pi+\gamma, \\ -i_{\mathrm{H}^{*}} & \text { при } & \pi+\gamma \leqslant \omega t \leqslant 2 \pi .\end{array}\right.$

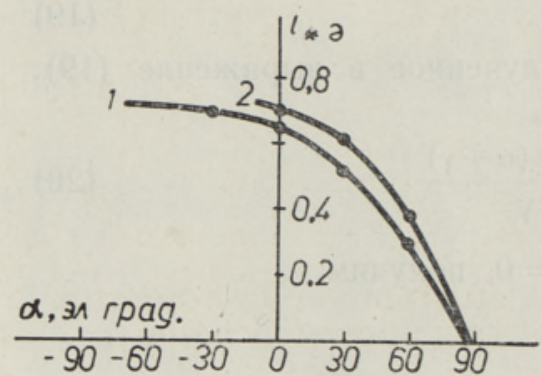

Рис. 4. Зависимость относительного расчетного действующего значения тока $i_{*}$ от угла регулирования $\alpha$ при $\Theta=10$ (1) и $80^{\circ}$ (2).

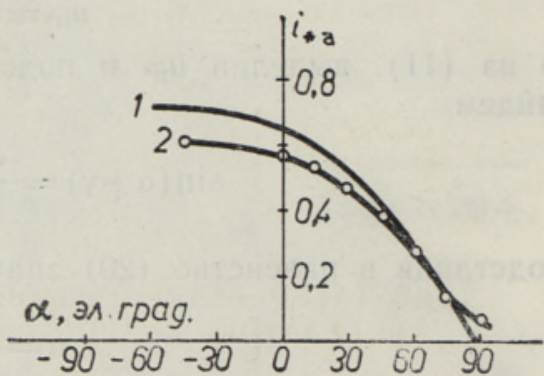

Рис. 5. Зависимость относительного действующего значения тока $i_{*}$ от угла регулирования $\alpha$ при $\Theta=30^{\circ}$ : расчетная кривая (1), опытная кривая (2).

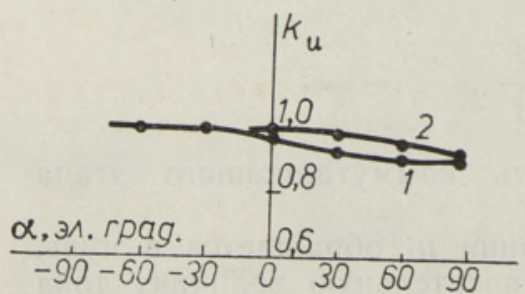

Рис. 6. Зависимость значений коэффициента искажения $k_{\text {n }}$ от угла регулирования $\alpha$ при $\Theta=10$ (1) и $80^{\circ}(2)$.
Из выражения (18) вытекает $\alpha_{\text {пр }}=0$. Зависимость между $\alpha$ и $\gamma$ имеет вид

$$
\gamma=\pi-2 \alpha \text {. }
$$

Подставив равенство (26) в формулу (22), получим $i_{\mathrm{H}^{*}}=\cos \alpha$.

На основе выражений (14)-(16) и (21) были рассчитаны регулировочные характеристики, отражающие зависимость относительного действующего значения тока $i_{*}$ от угла $\alpha$. Эти 
характеристики показаны на рис. 4 при значениях угла нагрузки $\Theta=10$ и $80^{\circ}$. На рис. 5 приведены расчетная и опытная регулировочные характеристики при $\Theta=30^{\circ}$. Видно, что при $\alpha=90$ эл. град. значение $i *$ на опытной кривой не равно нулю. Причиной этого является конечное значение индуктивности дросселя Д. С уменьшением угла $\alpha$ отклонение опытной кривой от расчетной увеличивается, что объясняется все возрастающим влиянием активного сопротивления коммутационного контура, которое при выводе расчетных формул не учитывалось. Кривые изменения коэффициента искажения при $\Theta=10$ и $80^{\circ}$ в зависимости от $\boldsymbol{\alpha}$ изображены на рис. 6.

На основе изложенного выше можно заключить, что переход от активной нагрузки к активно-индуктивной не препятствует нормальной работе тиристорного регулятора переменного напряжения с подавленными четными гармониками и способствует дальнейшему подавлению высших гармоник в токе регулятора.

\section{Л И Т Е Р А Т У Р А}

1. Em a nuel-Eigeles, A., Erlicki, M. S., IEEE Trans. Industry and General Applications, IGA-4, No. 4, 447 (1968).

2. Le p p, V. R., S i b g a tuli n, K. M., C he rk a s ov, I. N., Current stabilizer having a saturable reactor in the mode of forced magnetization, United States Patent No. 3845380 , Oct. $29,1974$.

3. О я в э р М., Сар в В., Изв. АН ЭССР, Физ. Матем., 25, 392 (1976).

4. Т е х в е Х., Изв. АН ЭССР, Физ. Матем., 26, 186 (1977).

5. Булгаков А. А., Новая теория управляемых выпрямителей, М., 1970.

Институт термофизики и электрофизики Академии наук Эстонской ССР
Поступнла в редакцию 2/XI 1976

\section{G. BATARIN}

\section{TÓKESTATUD PAARISHARMOONILISTEGA VAHELDUVPINGE-TORISTOR- REGULAATORI ANALOUS AKTIIV-INDUKTIIVKOORMUSE KORRAL}

Näidatakse, et koormuse induktiivkomponent soodustab koormusvoolu nõrgemate harmooniliste vähendamist. On leitud koormusvoolu suhtelise efektiivväärtuse ning kujuieguri sõltuvus reguleerimisnurgast ja nihkenurgast.

\section{G. BATARIN}

\section{ANALYSIS OF $A-C$ VOLTAGE SCR REGULATOR WITH SUPPRESSED EVEN HARMONICS IN THE CASE OF RESISTIVE-INDUCTIVE LOAD}

Operation of the $a-c$ voltage SCR regulator with suppressed even harmonics in the case of resistive-inductive load is analyzed. Expressions of both relative effective value of load current and form factor in dependence on the values of the load angle are presented. 